\title{
Post-exertional thoracic pseudomeningocoele in a 41-year-old woman: a rare cause of interscapular pain
}

\author{
J R Ouma, S Makhure \\ Department of Neurological Surgery, University of the Witwatersrand, Johannesburg and Chris Hani Baragwanath Academic \\ Hospital, Soweto.
}

Corresponding author: Dr. John R. Ouma (glioma42@gmail.com)

\begin{abstract}
Summary: We present an unusual case of a previously healthy 41-year-old lady who acutely developed severe, disabling interscapular pain while lifting heavy boxes. She had no associated neurological deficits.

She was found to have dorsal thoracic pseudomeningocoeles on magnetic resonance imaging (MRI) that were successfully managed surgically.

While post-exertional spine pain is a common occurrence in every day clinical practice, this is often due to sprains and strains of muscles, tendons and ligaments. In a few cases, imaging reveals lesions such as annular disc tears, disc protrusions, or fractures of osteoporotic vertebra.

Pseudomeningocoeles are generally described as complicating surgery or trauma to the spine. This case is unique as it follows exertional stress, which, to our knowledge, is not commonly described in the literature. Further, she had no risk factors such as connective tissue disorders that might have predisposed her to this development.
\end{abstract}

\section{Introduction}

We present an interesting case of a 41-year-old lady who works as an administrative assistant in a local high school.

She reports that while lifting heavy boxes some six months ago, she developed sudden and severe interscapular pain. Her symptoms had persisted and worsened over the period in spite of the best efforts of her local doctors. She denied any pain or weakness in any of her limbs. Her past medical history was unremarkable for any trauma or significant medical conditions. Clinically, she was in severe pain, and walked in a stooped fashion. She had exquisite tenderness in the upper dorsal spine area, but no deformity. Neurological examination was unremarkable.

$\mathrm{X}$-rays of the dorsal spine were negative; however, a magnetic resonance imaging (MRI) scan of the area demonstrated the presence of two distinct, fluid filled dorsal extradural lesions at the T3 and T4 level (Figures 1 and 2).

The differential diagnoses being considered by the radiologists included parasitic disease, neoplasms and infections, none of which seemed to tally with the history as given by the patient.

In theatre, two distinct masses containing cerebrospinal fluid (CSF) were found, enclosed within a fibrous capsule. On opening the capsules, there was CSF egress and dural defects were visible at both sites which were repaired in the standard fashion. There were no neural elements herniating into the sacs, and the walls were sent for histology which demonstrated arachnoid enmeshed in a fibrous capsule.

A diagnosis of thoracic pseudomeningocoele was hence established.

Post-operatively she did very well indeed with immediate resolution of her pain.

\section{Discussion}

Pseudomeningocoeles are so called to differentiate them from true meningocoeles.

In the former, CSF escapes from the subarachnoid space to lie in the extradural space, and in time becomes covered by a false capsule or in some instances an intact arachnoid layer, ${ }^{1}$ while in the latter all layers of the meninges are present in the sac.

Pseudomeningocoeles usually form in the setting of trauma or as a complication of intentional or accidental durotomy at surgery, ${ }^{1-6}$ while true meningocoeles occur in the setting of spinal dysraphism.

It is reported that the diagnosis of pseudomeningocoeles is not always straightforward and a number of them are asymptomatic. ${ }^{1}$ 

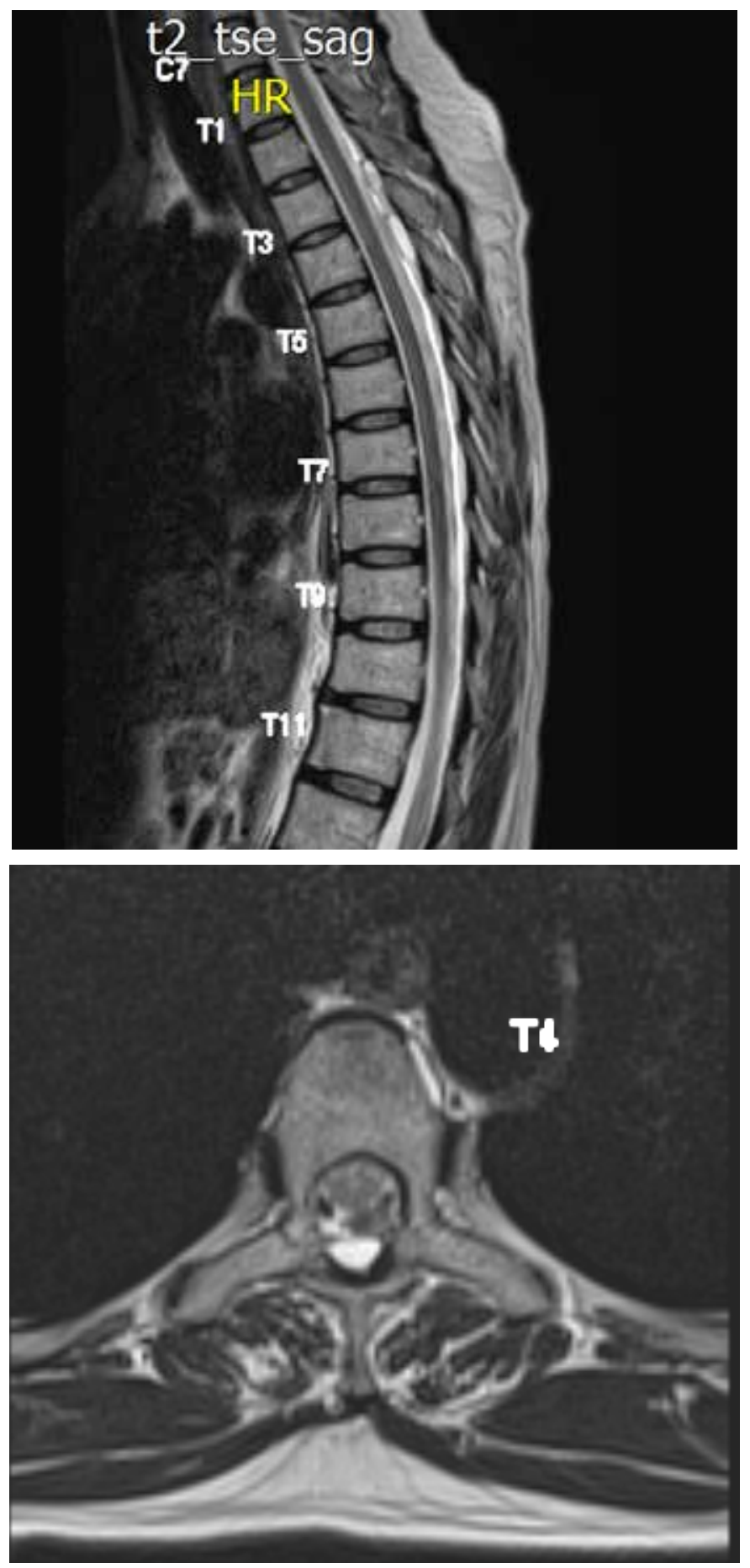

Figures 1 and 2: Sagittal T1 and T2 Magnetic resonance imaging (MRI) scans respectively showing dorsal, cyst like lesions at the T3 and T4 levels

A history of spinal trauma or else surgery to the spine is helpful in increasing the index of suspicion for the condition, and this should always be considered in the appropriate clinical setting. ${ }^{1,2,4-7}$

Where symptoms do occur, these have been ascribed to the sheer size that some of these lesions can achieve, or else neurological deficits occasioned by herniation into or compression of nerve roots and the spinal cord into the sac., ${ }^{2,-7}$
The management of symptomatic psedomeningocoeles of the spine is generally by direct exposure of the lesion, removal of the cyst wall and repair of the dural defect., ${ }^{1,2}$ Some large ones have been managed indirectly by CSF diversion shunts, ${ }^{6}$ while there are reports of spontaneous resolution of pseudomeningocoeles. ${ }^{8,9}$

This case report is unique in that there is hardly any mention in the literature of this condition occurring in the setting of physical exertion. While sudden spinal pain following physical exertion is common in clinical practice, it is mainly encountered in the lumbar spine and where sprains and strains have been excluded, it is usually due to annular tears of intervertebral discs or else, in a few cases, disc prolapses or fractures of osteoporotic vertebra in our experience. While pseudomeningocoeles have been reported to occur in conditions such as neurofibromatosis and Marfans syndrome, in fact the majority of these have been found to be true meningocoeles. ${ }^{10}$ This lady had no such conditions.

There are case reports in the literature of intracranial hypotension occurring in the setting of spontaneous spinal CSF leaks. In most cases, the patients are females, and present with orthostatic headaches, as well as nausea, vomiting and even abducens cranial nerve palsies. ${ }^{11,12}$ It is interesting to note that this patient did not have any of these symptoms.

This publication alerts spine surgeons as to this rare condition, which should be included in the differential diagnosis of acute and severe spinal pain following physical exertion.

As demonstrated in our case report, while the pain and discomfort were excruciating, the management was relatively straightforward and the outcome rewarding for patient and surgeon alike.

\section{Teaching points}

1. Spinal surgeons must be aware of spinal pseudomeningocoeles presenting in unusual fashions and should have a high index of suspicion for these lesions in appropriate circumstances.

2. Careful consideration of investigations such as MRI scans is essential for timeous diagnosis of pathology.

3. This condition lends itself to effective treatment once diagnosed correctly.

\section{REFERENCES}

1. Julia PE, Nazirah H. Spinal pseudomeningocoele: a diagnostic dilemma. Spinal cord. 2007;45(12):804-5.

2. Aiyer SN, Shetty AP, Kanna R, Maheswaran A, Rajasekaran S. Spinal cord herniation following cervical meningioma excision: a rare clinical entity and review of literature. European spine journal: official publication of the European Spine Society, the European Spinal Deformity Society, and the European Section of the Cervical Spine Research Society. 2016;25(Suppl 1):2169.

3. Fan YF, Chong VF. MRI findings in failed back surgery syndrome. Med J Malaysia. 1995;50(1):76-81.

4. Johnson DB, McGrath FP. Case report: post-traumatic thoracolumbar pseudomeningocoele--an unusual cause of upper 
lumbar pain. Clin Radiol. 1997;52(9):715-7.

5. Phillips PM, Correia JA, Karlsson PM, Muthu T, Balakrishnan V, Hussain Z. Symptomatic delayed trans-dural herniation and strangulation of cauda equinae following resection of Myxopapillary ependymoma. Br J Neurosurg. 2012;26(3):4323 .

6. Singh M, Kasliwal MK, Mahapatra AK. Giant cervical pseudomeningocoele following brachial plexus trauma. J Clin Neurosci. 2008;15(3):310-3.

7. Pavlou G, Bucur SD, van Hille PT. Entrapped spinal nerve roots in a pseudomeningocoele as a complication of previous spinal surgery. Acta Neurochirurgica. 2006;148(2):215-9; discussion 9-20.
8. Clarke A, Hutton M. Spontaneous resolution of a massive pseudomeningocoele. Acta orthopaedica Belgica. 2009;75(2):277-9.

9. Kumar AJ, Nambiar CS, Kanse P. Spontaneous resolution of lumbar pseudomeningocoele. Spinal cord. 2003;41(8):470-2.

10. Pyeritz RE: The Marfan syndrome. Am Fam Physician 1986.34: 83-94.

11. Schievink W. Spontaneous spinal cerebrospinal fluid leaks and intracranial hypotension. JAMA 2006;295(19):2286-96.

12. Rando T, Fishman R. Spontaneous intracranial hypotension. Report of two cases and review of the literature. Neurology. March 1992;42(3):481. 